1 Hacettepe Journal of Mathematics and Statistics

$\bigcap$ Volume 43 (1) (2014), 27-41

\title{
ON A FUNCTIONAL EQUATION ORIGINATING FROM A MIXED ADDITIVE AND CUBIC EQUATION AND ITS STABILITY
}

\author{
Mohammad Janfada $^{\mathrm{a} *}$, Tayebe Laal Shateri ${ }^{\mathrm{b}}{ }^{\dagger}$, and Rahele Shourvarzi ${ }^{\mathrm{c}}{ }^{\ddagger}$
}

Received $07: 08: 2011$ : Accepted 25:12:2012

\begin{abstract}
In this paper, we study solutions of the 2-variable mixed additive and cubic functional equation

$$
\begin{aligned}
f(2 x+y, 2 z+t)+ & f(2 x-y, 2 z-t)=2 f(x+y, z+t) \\
+ & 2 f(x-y, z-t)+2 f(2 x, 2 z)-4 f(x, z),
\end{aligned}
$$

which has the cubic form $f(x, y)=a x^{3}+b x^{2} y+c x y^{2}+d y^{3}$ as a solution. Also the Hyers-Ulam-Rassias stability of this equation in the non-Archimedean Banach spaces is investigated.
\end{abstract}

Keywords: Hyers-Ulam-Rassias stability, Cubic functional equation, Non-Archimedean normed space, Derivation.

2000 AMS Classification: 39B22, 39B82, 46S10

\section{Introduction and preliminaries}

The study of stability problems for functional equations is related to a question of Ulam [26] concerning the stability of group homomorphisms, affirmatively answered for Banach spaces by Hyers [8]. Subsequently, the result of Hyers was generalized by Aoki [1], Bourgin [5] and Rassias [24].

During the last decades several stability problems for various functional equations have been investigated by numerous mathematicians. We refer the reader to the survey articles $[7,9,23]$ and monographs $[6,10,13,22]$ and references therein.

${ }^{\mathrm{a} D e p a r t m e n t}$ of Mathematics, Ferdowsi University of Mashhad, Mashhad, P. O. Box 115991775 Iran.

*Email: mjanfada@gmail.com

${ }^{\mathrm{b}}$ Facaulty of Mathematics and computer sciences, Hakim Sabzevari University, Sabzevar, Iran.

†Email: t.shateri@gmail.com

${ }^{\mathrm{c}}$ Department of Mathematics, Hakim Sabzevari University, Sabzevar, Iran.

${ }^{\ddagger}$ Email: rshurvarzy@gmail.com 
Let $\mathrm{X}$ and $\mathrm{Y}$ be real vector spaces. For a mapping $f: X \times X \rightarrow Y$, consider the following 2-variable mixed additive and cubic functional equation:

$$
\begin{aligned}
f(2 x+y, 2 z+t)+f(2 x-y, 2 z-t)=2 f(x+y, z+t) & +2 f(x-y, z-t) \\
& +2 f(2 x, 2 z)-4 f(x, z)
\end{aligned}
$$

One can see that the cubic form $f(x, y)=a x^{3}+b x^{2} y+c x y^{2}+d y^{3}$ is a solution of (1.1), when $X=Y=\mathbb{R}$.

The one variable cubic equation

$$
f(2 x+y)+f(2 x-y)=2 f(x+y)+2 f(x-y)+2 f(2 x)-4 f(x)
$$

is considered in [17] and the general solutions of this equation and its Hyers-Ulam-Rassias stability in quasi-Banach spaces is studied.

Several-variable functional equations and their stability have been studied in many papers (see, for example, [3, 4], [12, 11], [14], [18, 19], [20, 21], [25]).

In this paper first we study solutions of (1.1) and its relations with (1.2) and then the Hyers-Ulam-Rassias stability of (1.1) in non-Archimedean Banach spaces is investigated.

By a non-Archimedean field we mean a field $\mathbb{K}$ equipped with a function (valuation) $|\cdot|$ from $\mathbb{K}$ into $[0, \infty)$ such that $|r|=0$ if and only if $r=0,|r s|=|r||s|$, and $|r+s| \leq$ $\max \{|r|,|s|\}$ for all $r, s \in \mathbb{K}$. Clearly $|1|=|-1|=1$ and $|n| \leq 1$ for all $n \in \mathbb{N}$. By the trivial valuation we mean the mapping $|\cdot|$ taking everything but 0 into 1 and $|0|=0$. Let $X$ be a vector space over a field $\mathbb{K}$ with a non-Archimedean non-trivial valuation $|\cdot|$. A function $\|\cdot\|: X \rightarrow[0, \infty)$ is called a non-Archimedean norm if it satisfies the following conditions:

(i) $\|x\|=0$ if and only if $x=0$;

(ii) for any $r \in \mathbb{K}, x \in X,\|r x\|=|r|\|x\|$;

(iii) the strong triangle inequality (ultrametric); namely,

$$
\|x+y\| \leq \max \{\|x\|,\|y\|\} \quad(x, y \in X) .
$$

Then $(X,\|\cdot\|)$ is called a non-Archimedean normed space. Due to the fact that

$$
\left\|x_{n}-x_{m}\right\| \leq \max \left\{\left\|x_{j+1}-x_{j}\right\|: m \leq j \leq n-1\right\} \quad(n>m),
$$

a sequence $\left\{x_{n}\right\}$ is Cauchy if and only if $\left\{x_{n+1}-x_{n}\right\}$ converges to zero in a nonArchimedean normed space. By a complete non-Archimedean normed space we mean one in which every Cauchy sequence is convergent.

The stability problem in non-Archimedean normed spaces has been studied by many authors. In [2], the stability of approximate additive mappings $f: \mathbb{Q}_{p} \rightarrow \mathbb{R}$ is investigated. In $[15,16]$, the authors investigated the stability of Cauchy, quadratic and cubic functional equations, in the context of non-Archimedean normed spaces.

We need the following lemmas from [17] for our stability results.

1.1. Lemma. If a mapping $f: X \rightarrow Y$ with $f(0)=0$ satisfies (1.2), then the mapping $g: X \rightarrow Y$ defined by $g(x)=f(2 x)-8 f(x)$ is additive.

1.2. Lemma. If a mapping $f: X \rightarrow Y$ with $f(0)=0$ satisfies (1.2), then the mapping $h: X \rightarrow Y$ defined by $h(x)=f(2 x)-2 f(x)$ is cubic.

\section{Relations between (1.2) and (1.1)}

In this section we show that equations (1.2) and (1.1) are closely related and so by knowing the solutions of (1.2), we may find solutions of (1.1). Next some useful examples are considered. 
2.1. Theorem. Suppose $f: X \times X \rightarrow Y$ is a mapping satisfying (1.1), then $g: X \rightarrow Y$ defined by $g(x):=f(x, x)$ satisfies (1.2).

Proof. From (1.1) and definition of $g$,

$$
\begin{aligned}
g(2 x+y)+g(2 x-y) & =f(2 x+y, 2 x+y)+f(2 x-y, 2 x-y) \\
& =2 f(x+y, x+y)+2 f(x-y, x-y)+2 f(2 x, 2 x)-4 f(x, x) \\
& =2 g(x+y)+2 g(x-y)+2 g(2 x)-4 g(x) .
\end{aligned}
$$

2.2. Theorem. Let $a, b, c, d \in \mathbb{R}$ and $g: X \rightarrow Y$ be a mapping satisfying (1.2). If $f: X \times X \rightarrow Y$ is defined by

$$
f(x, y)=\left(a-\frac{c}{3}\right) g(x)+\left(\frac{c+b}{6}\right) g(x+y)+\left(\frac{c-b}{6}\right) g(x-y)+\left(d-\frac{b}{3}\right) g(y),
$$

then $f$ satisfies (1.1). Furthermore if $f(0,0)=0, a+d=1$ and $c=-b$, then $g(x)=$ $f(x, x)$.

Proof. We have

$$
\begin{aligned}
f(2 x+y, 2 z+t)+f(2 x-y, 2 z-t) & =\left(a-\frac{c}{3}\right) g(2 x+y)+\left(\frac{c+b}{6}\right) g(2 x+y+2 z+t) \\
& +\left(\frac{c-b}{6}\right) g(2 x+y-(2 z+t))+\left(d-\frac{b}{3}\right) g(2 z+t) \\
& +\left(a-\frac{c}{3}\right) g(2 x-y)+\left(\frac{c+b}{6}\right) g(2 x-y+2 z-t) \\
& +\left(\frac{c-b}{6}\right) g(2 x-y-(2 z-t))+\left(d-\frac{b}{3}\right) g(2 z-t) \\
& =\left(a-\frac{c}{3}\right)[g(2 x+y)+g(2 x-y)] \\
& +\left(\frac{c+b}{6}\right)[g(2 x+2 z y+t)+g(2 x+2 z-(y+t))] \\
& +\left(\frac{c-b}{6}\right)[g(2 x-2 z+y-t)+g(2 x-2 z-(y-t))] \\
& +\left(d-\frac{b}{3}\right)[g(2 z+t)+g(2 z-t)] .
\end{aligned}
$$

On the other hand

$$
\begin{aligned}
& 2 f(x+y, z+t)+2 f(x-y, z-t)+2 f(2 x, 2 z)-4 f(x, z) \\
= & \left(a-\frac{c}{3}\right)[2 g(x+y)+2 g(x-y)+2 g(2 x)-4 g(x)] \\
+ & \left(\frac{c+b}{6}\right)[2 g(x+y+z+t)+2 g(x+z-(y+t))+2 g(2 x+2 z)-4 g(x+z)] \\
+ & \left(\frac{c-b}{6}\right)[2 g(x-z+y-t)+2 g(x-z-(y-t))+2 g(2 x-2 z)-4 g(x-z)] \\
+ & \left(d-\frac{b}{3}\right)[2 g(z+t)+2 g(z-t)+2 g(2 z)-4 g(z)] \\
= & \left(a-\frac{c}{3}\right)[g(2 x+y)+g(2 x-y)] \\
+ & \left(\frac{c+b}{6}\right)[g(2 x+2 z y+t)+g(2 x+2 z-(y+t))] \\
+ & \left(\frac{c-b}{6}\right)[g(2 x-2 z+y-t)+g(2 x-2 z-(y-t))] \\
+ & \left(d-\frac{b}{3}\right)[g(2 z+t)+g(2 z-t)] .
\end{aligned}
$$


Thus (2.2) and (2.3) imply that f satisfies (1.1).

For the following example, we recall that a mapping $D$ from an algebra $X$ into itself is called derivation if, for any $x, y \in X, D(x y)=D(x) y+x D(y)$.

2.3. Example. Let $X$ be a real algebra and let $D_{1}$ be a derivation on $X$. Suppose $D_{2}: X \rightarrow X$ satisfies

$$
D_{2}(x y)=D_{2}(x) y+D_{1}(x) D_{1}(y)+x D_{2}(y) .
$$

Now define $f: X \times X \rightarrow X$ by $f(x, y)=D_{2}(x y)$, then $f$ satisfies (1.1). Also $g: X \rightarrow X$ defined by $g(x)=D_{2}\left(x^{2}\right)$ satisfies (1.2).

2.4. Example. Let $M_{n}$ be the algebra of $n \times n$-real matrices. Define the mapping $g: M_{n} \rightarrow M_{n}$ by $g(A)=A^{3}, A \in M_{n}$, then one can easily see that $g$ satisfies (1.2). For $a, b, c, d \in \mathbb{R}$, set

$$
f(A, B)=a A^{3}+\frac{2 b}{3} A^{2} o B+\frac{2 c}{3} A o B^{2}+\frac{b}{3} A B A+\frac{b}{3} B A B+d B A B,
$$

where $A o B$ is the Jordan product $\frac{1}{2}(A B+B A)$ of $A$ and $B$, for any $A, B \in M_{n}$. Then $f$ satisfies (2.1). So by Theorem $2.2, f$ satisfies (1.1).

\section{Stability of Eq. (1.2)}

Throughout this section, assume that $X$ is a vector space and that $Y$ is a nonArchimedean Banach space. In this section, we study some stability results from [17] in non-Archimedean Banach spaces. Indeed, we consider the stability of functional equation (1.1), and the fact the $X \times X$ with the point-wise operations is also a vector space implies a similar stability result for (1.2). For convenience, we use the following abbreviation for a given mapping $f: X \rightarrow Y$,

$$
D f(x, y):=f(2 x+y)+f(2 x-y)-2 f(x+y)-2 f(x-y)-2 f(2 x)+4 f(x)
$$

for all $x, y \in X$.

3.1. Theorem. Let $\varphi_{a}: X \times X \rightarrow[0, \infty)$ be a mapping such that

$$
\begin{aligned}
& \lim _{n \rightarrow \infty} \frac{1}{|2|^{n}} \varphi_{a}\left(2^{n} x, 2^{n} y\right)=0 \\
& M_{a}(x, y):=\lim _{n \rightarrow \infty} \max \left\{\frac{1}{|2|^{i}} \varphi_{a}\left(2^{i} x, 2^{i} y\right): 0 \leq i<n\right\}<\infty \\
& \lim _{t \rightarrow \infty} \lim _{n \rightarrow \infty} \max \left\{\frac{1}{|2|^{i}} \varphi_{a}\left(2^{i} x, 2^{i} y\right): t \leq i<t+n\right\}=0
\end{aligned}
$$

for all $y \in X$ and all $x \in\left\{0, y, \frac{y}{2}\right\}$. Suppose that a mapping $f: X \rightarrow Y$ with $f(0)=0$ satisfies the inequality

$$
\|D f(x, y)\|_{Y} \leq \varphi_{a}(x, y)
$$

for all $x, y \in X$. Then the limit

$$
A(x)=\lim _{n \rightarrow \infty} \frac{1}{2^{n}}\left[f\left(2^{n+1} x\right)-8 f\left(2^{n} x\right)\right]
$$

exists, for all $x \in X$, and the mapping $A: X \rightarrow Y$ is the unique additive mapping satisfying

$$
\|f(2 x)-8 f(x)-A(x)\|_{Y} \leq \frac{1}{|2|} \tilde{\varphi}_{a}(x),
$$

for all $x \in X$, where

$$
\tilde{\varphi}_{a}(x):=\max \left\{|2| M_{a}(x, x),|2| M_{a}(0, x), M_{a}(x, 2 x)\right\}
$$


Proof. Letting $x=0$ in (3.4), we get

$$
\|f(y)+f(-y)\|_{Y} \leq \varphi_{a}(0, y)
$$

for all $y \in X$. Replacing $y$ by $x$ and $2 x$ in (3.4), respectively, we get the following inequalities

$$
\begin{aligned}
& \|f(3 x)-4 f(2 x)+5 f(x)\|_{Y} \leq \varphi_{a}(x, x) \\
& \|f(4 x)-2 f(3 x)-2 f(2 x)-2 f(-x)+4 f(x)\|_{Y} \leq \varphi_{a}(x, 2 x)
\end{aligned}
$$

for all $x \in X$. It follows from (3.6)-(3.8) that for any $x \in X$,

$$
\|f(4 x)-10 f(2 x)+16 f(x)\|_{Y} \leq \max \left\{|2| \varphi_{a}(x, x),|2| \varphi_{a}(0, x), \varphi_{a}(x, 2 x)\right\} .
$$

Let $g: X \rightarrow Y$ be a mapping defined by $g(x):=f(2 x)-8 f(x)$ and let

$$
\psi_{a}(x):=\max \left\{|2| \varphi_{a}(x, x),|2| \varphi_{a}(0, x), \varphi_{a}(x, 2 x)\right\},
$$

for all $x \in X$. Therefore (3.9) means

$$
\|g(2 x)-2 g(x)\|_{Y} \leq \psi_{a}(x),
$$

for all $x \in X$. By relations (3.1)-(3.3) we infer that for all $x \in X$,

$$
\max \left\{\frac{\psi_{a}\left(2^{i} x\right)}{|2|^{i}}: 0 \leq i<n\right\}<\infty, \quad \lim _{n \rightarrow \infty} \frac{1}{|2|^{n}} \psi_{a}\left(2^{n} x\right)=0 .
$$

Replacing $x$ by $2^{n} x$ in (3.10) and dividing both sides (3.10) by $|2|^{n+1}$ we get

$$
\left\|\frac{1}{2^{n+1}} g\left(2^{n+1} x\right)-\frac{1}{2^{n}} g\left(2^{n} x\right)\right\|_{Y} \leq \frac{1}{|2|^{n+1}} \psi_{a}\left(2^{n} x\right)
$$

for all $x \in X$ and all non-negative integer $n$, and so for any $x \in X$ and every non-negative integers $n$ and $m$ with $n \geq m$,

$$
\left\|\frac{1}{2^{n}} g\left(2^{n} x\right)-\frac{1}{2^{m}} g\left(2^{m} x\right)\right\|_{Y} \leq \frac{1}{|2|} \max \left\{\frac{\psi_{a}\left(2^{i} x\right)}{|2|^{i}}: m \leq i<n\right\} .
$$

Therefore we conclude from (3.11) and (3.12) that the sequence $\left\{\frac{1}{2^{n}} g\left(2^{n} x\right)\right\}$ is a Cauchy sequence in $Y$, for all $x \in X$. The sequence $\left\{\frac{1}{2^{n}} g\left(2^{n} x\right)\right\}$ converges in $Y$ for any $x \in X$, since $Y$ is complete. So one can define the mapping $A: X \rightarrow Y$ by

$$
A(x):=\lim _{n \rightarrow \infty} \frac{1}{2^{n}} g\left(2^{n} x\right)
$$

for all $x \in X$. Letting $m=0$ and passing to the limit when $n \rightarrow \infty$ in (3.13), we get (3.5). Now we show that $A$ is an additive mapping. It follows from (3.11), (3.12) and (3.14) that

$$
\begin{aligned}
\|A(2 x)-2 A(x)\|_{Y} & =\lim _{n \rightarrow \infty}\left\|\frac{1}{2^{n}} g\left(2^{n+1} x\right)-\frac{1}{2^{n-1}} g\left(2^{n} x\right)\right\|_{Y} \\
& =|2| \lim _{n \rightarrow \infty}\left\|\frac{1}{2^{n+1}} g\left(2^{n+1} x\right)-\frac{1}{2^{n}} g\left(2^{n} x\right)\right\|_{Y} \\
& \leq \lim _{n \rightarrow \infty} \frac{1}{|2|^{n}} \psi_{a}\left(2^{n} x\right),
\end{aligned}
$$

for all $x \in X$. So

$$
A(2 x)=2 A(x)
$$


for all $x \in X$. On the other hand it follows from (3.1), (3.4) and (3.14) that

$$
\begin{aligned}
\|D A(x, y)\|_{Y} & =\lim _{n \rightarrow \infty} \frac{1}{|2|^{n}}\left\|D g\left(2^{n} x, 2^{n} y\right)\right\|_{Y} \\
& =\lim _{n \rightarrow \infty} \frac{1}{|2|^{n}}\left\{\left\|D f\left(2^{n+1} x, 2^{n+1} y\right)-8 D f\left(2^{n} x, 2^{n} y\right)\right\|_{Y}\right\} \\
& \leq \lim _{n \rightarrow \infty} \frac{1}{|2|^{n}} \max \left\{\left\|D f\left(2^{n+1} x, 2^{n+1} y\right)\right\|_{Y},|8|\left\|D f\left(2^{n} x, 2^{n} y\right)\right\|_{Y}\right\} \\
& \leq \lim _{n \rightarrow \infty} \frac{1}{|2|^{n}} \max \left\{\varphi_{a}\left(2^{n+1} x, 2^{n+1} y\right),|8| \varphi_{a}\left(2^{n} x, 2^{n} y\right)\right\}=0,
\end{aligned}
$$

for all $x, y \in X$. Hence the mapping $A$ satisfies (1.2). So by Lemma 1.1, the mapping $x \mapsto A(2 x)-8 A(x)$ is additive. Therefore (3.15) implies that $A$ is additive. To prove the uniqueness of $A$, let $T: X \rightarrow Y$ be another additive mapping satisfying (3.5). So it follows from (3.5), (3.14) and (3.3) that

$$
\begin{aligned}
\lim _{t \rightarrow \infty} \frac{1}{|2|^{t}} \tilde{\varphi}_{a}\left(2^{t x}\right)= & \lim _{t \rightarrow \infty} \max \left\{|2| \frac{M_{a}\left(2^{t} x, 2^{t} x\right)}{|2|^{t}},|2| \frac{M_{a}\left(0,2^{t} x\right)}{|2|^{t}}, \frac{M_{a}\left(2^{t} x, 2^{t} 2 x\right)}{\left.|2|^{t}\right\}}\right. \\
= & \lim _{t \rightarrow \infty} \max \left\{\lim _{n \rightarrow \infty} \max \left\{|2| \frac{\varphi_{a}\left(2^{i+t} x, 2^{i+t} x\right)}{|2|^{t+i)}}: 0 \leq i<n\right\},\right. \\
& \lim _{n \rightarrow \infty} \max \left\{|2| \frac{\varphi_{a}\left(0,2^{i+t} x\right)}{|2|^{t+i}} 0 \leq i<n\right\}, \\
& \lim _{n \rightarrow \infty} \max \left\{\frac{\varphi_{a}\left(2^{i+t} x, 2^{i+t+1} x\right)}{\left.\left.|2|^{t+i)}: 0 \leq i<n\right\}\right\}}\right. \\
= & \lim _{t \rightarrow \infty} \lim _{n \rightarrow \infty}\left\{|2| \frac{\varphi_{a}\left(2^{i} x, 2^{i} x\right)}{|2|^{i}},|2| \frac{\varphi_{a}\left(0,2^{i} x\right)}{|2|^{i}}, \frac{\varphi_{a}\left(2^{i} x, 2^{i+1} x\right)}{|2|^{i}}: t \leq i<t+n\right\} \\
= & 0 .
\end{aligned}
$$

Hence it follows

$$
\begin{aligned}
\|A(x)-T(x)\|_{Y} & =\lim _{t \rightarrow \infty} \frac{1}{|2|^{t}}\left\|g\left(2^{t} x\right)-T\left(2^{t} x\right)\right\|_{Y} \\
& \leq \frac{1}{|2|} \lim _{t \rightarrow \infty} \frac{1}{|2|^{t}} \tilde{\varphi}_{a}\left(2^{t x}\right)=0
\end{aligned}
$$

for all $x \in X$. So $A=T$

3.2. Theorem. Let $\varphi_{a}: X \times X \rightarrow[0, \infty)$ be a mapping such that

$$
\begin{aligned}
& \lim _{n \rightarrow \infty}|2|^{n} \varphi_{a}\left(\frac{x}{2^{n}}, \frac{y}{2^{n}}\right)=0, \\
& M_{a}(x, y)=\lim _{n \rightarrow \infty} \max \left\{|2|^{i} \varphi_{a}\left(\frac{x}{2^{i}}, \frac{y}{2^{i}}\right): 1 \leq i<n\right\}<\infty, \\
& \lim _{t \rightarrow \infty} \lim _{n \rightarrow \infty} \max \left\{|2|^{i} \varphi_{a}\left(\frac{x}{2^{i}}, \frac{y}{2^{i}}\right): t+1 \leq i<t+n\right\}=0,
\end{aligned}
$$

for all $y \in X$ and all $x \in\left\{0, y, \frac{y}{2}\right\}$. Suppose that a mapping $f: X \rightarrow Y$ with $f(0)=0$ satisfies the inequality

$$
\|D f(x, y)\|_{Y} \leq \varphi_{a}(x, y)
$$

for all $x, y \in X$. Then the limit

$$
A(x)=\lim _{n \rightarrow \infty} 2^{n}\left[f\left(\frac{x}{2^{n-1}}\right)-8 f\left(\frac{x}{2^{n}}\right)\right]
$$

exists for all $x \in X$ and the mapping $A: X \rightarrow Y$ is the unique additive mapping satisfying

$$
\|f(2 x)-8 f(x)-A(x)\|_{Y} \leq \frac{1}{|2|} \tilde{\varphi}_{a}(x)
$$


for all $x \in X$, where

$$
\tilde{\varphi}_{a}(x):=\max \left\{|2| M_{a}(x, x),|2| M_{a}(0, x), M_{a}(x, 2 x)\right\} .
$$

Proof. Let $g: X \rightarrow Y$ be a mapping defined by $g(x):=f(2 x)-8 f(x)$ and let

$$
\psi_{a}(x):=\max \left\{|2| \varphi_{a}(x, x),|2| \varphi_{a}(0, x), \varphi_{a}(x, 2 x)\right\},
$$

for all $x \in X$. Similar to the proof of Theorem 3.1, we have

$$
\|g(2 x)-2 g(x)\|_{Y} \leq \psi_{a}(x),
$$

for all $x \in X$. From our assumptions, we infer that

$$
\lim _{n \rightarrow \infty}|2|^{n} \psi_{a}\left(\frac{x}{2^{n}}\right)=0, \quad \lim _{n \rightarrow \infty} \max \left\{|2|^{i} \psi_{a}\left(\frac{x}{2^{i}}\right): \quad 1 \leq i<n\right\}<\infty,
$$

for all $x \in X$. Replacing $x$ by $\frac{x}{2^{n+1}}$ in (3.19) and multiplying both sides of (3.19) by $|2|^{n}$, we get

$$
\left\|2^{n+1} g\left(\frac{x}{2^{n+1}}\right)-2^{n} g\left(\frac{x}{2^{n}}\right)\right\|_{y} \leq|2|^{n} \psi_{a}\left(\frac{x}{2^{n+1}}\right),
$$

for all $x \in X$ and all non-negative integer $n$. So we have

$$
\| 2^{n} g\left(\frac{x}{2^{n}}-2^{m} g\left(\frac{x}{2^{m}} \|_{Y} \leq \frac{1}{|2|} \max \left\{|2|^{(i+1)} \psi_{a}\left(\frac{x}{2^{i+1}}\right): \quad m \leq i<n\right\},\right.\right.
$$

for all $x \in X$ and all non-negative integers $n$ and $m$ with $n \geq m$. Therefore we conclude from (3.20) and (3.21) that the sequence $\left\{2^{n} g\left(\frac{x}{2^{n}}\right\}\right.$ is a Cauchy sequence in $Y$, for all $x \in X$ and so converges in $Y$, for all $x \in X$, since $Y$ is complete. Thus one can define the mapping $A: X \rightarrow Y$ by

$$
A(x):=\lim _{n \rightarrow \infty} 2^{n} g\left(\frac{x}{2^{n}}\right),
$$

for all $x \in X$. Letting $m=0$ and passing to the limit when $n \rightarrow \infty$ in (3.22) we get (3.18). The rest of the proof is similar to the proof of Theorem 3.1.

3.3. Corollary. Let $\theta, r, s$ be non-negative real numbers such that $r, s>1$ or $0 \leq r, s<1$ and $|2|<1$. Suppose that a function $f: X \rightarrow Y$ with $f(0)=0$ satisfies the inequality

$$
\|D f(x, y)\|_{Y} \leq \varphi_{a}(x, y):= \begin{cases}\theta, & r=s=0 \\ \theta\|x\|_{X}^{r}, & r>0, s=0 \\ \theta\|y\|_{X}^{s}, & r=0, s>0 \\ \theta\left(\|x\|_{X}^{r}+\|y\|_{X}^{s}\right), & r, s>0\end{cases}
$$

for all $x, y \in X$. Then there exists a unique additive mapping $A: X \rightarrow Y$ satisfying

$$
\begin{aligned}
& \|f(2 x)-8 f(x)-A(x)\|_{Y} \leq \\
& \frac{\theta}{|2|} \begin{cases}1, & r=s=0 \\
\|x\|_{X}^{r}, & r>0, s=0 \\
|2|\|x\|_{X}^{s}, & r=0, s>0 \\
\max \left\{|2|\left(\|x\|_{X}^{r}+\|x\|_{X}^{s}\right),\left(\|x\|_{X}^{r}+\|2 x\|_{X}^{s}\right)\right\}, & r, s>0\end{cases}
\end{aligned}
$$

for all $x \in X$ where $r, s>1$ and satisfying

$$
\|f(2 x)-8 f(x)-A(x)\|_{Y} \leq \frac{\theta}{|2|} \begin{cases}|2|, & r=s=0 \\ \frac{|2|\|x\|_{X}^{r}}{|2| r}, & r>0, s=0 \\ \frac{|4|\|x\|_{X}^{s}}{|2| s}, & r=0, s>0 \\ |2|\left(\frac{\|x\|_{X}^{r}}{|2|^{r}}+\|x\|_{X}^{s}\right), & r, s>0\end{cases}
$$

for all $x \in X$ where $r, s<1$. 
Proof. The result follows by Theorem 3.1 when $0<r, s<1$, and by Theorem 3.2 when $r, s>1$.

The following corollary also can be deduced from Theorems 3.1 and 3.2.

3.4. Corollary. Let $\theta \geq 0$ and $r, s>0$ be non-negative real numbers such that $\lambda:=$ $r+s \neq 1$. Suppose that the function $f: X \rightarrow Y$ with $f(0)=0$ satisfies the inequality

$$
\|D f(x, y)\|_{Y} \leq \varphi_{a}(x, y):=\theta\|x\|_{X}^{r}\|y\|_{Y}^{s}
$$

for all $x, y \in X$. Then there exists a unique additive mapping $A: X \rightarrow Y$ satisfying the inequality

$$
\|f(2 x)-8 f(x)-A(x)\|_{Y} \leq \frac{W \theta}{|2|}\|x\|_{X}^{r}\|y\|_{Y}^{s}
$$

for all $x, y \in X$ when $\lambda>1$ with $W=\max \left\{|2|,|2|^{s}\right\}$, and satisfying

$$
\|f(2 x)-8 f(x)-A(x)\|_{Y} \leq \frac{|2| \theta}{|2|^{\lambda}}\|x\|_{X}^{r}\|y\|_{Y}^{s}
$$

for all $x, y \in X$ when $\lambda<1$.

3.5. Theorem. Let $\varphi_{c}: X \times X \rightarrow[0, \infty)$ be a mapping such that

$$
\begin{aligned}
& \lim _{n \rightarrow \infty} \frac{1}{|8|^{n}} \varphi_{c}\left(2^{n} x, 2^{n} y\right)=0 \\
& M_{c}(x, y)=\lim _{n \rightarrow \infty} \max \left\{\frac{1}{|8|^{i}} \varphi_{c}\left(2^{i} x, 2^{i} y\right): 0 \leq i<n\right\}<\infty \\
& \lim _{t \rightarrow \infty} \lim _{n \rightarrow \infty} \max \left\{\frac{1}{|8|^{i}} \varphi_{c}\left(2^{i} x, 2^{i} y\right): t \leq i<t+n\right\}=0
\end{aligned}
$$

for all $y \in X$ and all $x \in\left\{0, y, \frac{y}{2}\right\}$. Suppose that a mapping $f: X \rightarrow Y$ with $f(0)=0$ satisfies the inequality

$$
\|D f(x, y)\|_{Y} \leq \varphi_{c}(x, y)
$$

for all $x, y \in X$. Then the limit

$$
C(x):=\lim _{n \rightarrow \infty} \frac{1}{8^{n}}\left[f\left(2^{n+1} x\right)-2 f\left(2^{n} x\right)\right]
$$

exists, for all $x \in X$, and the mapping $C: X \rightarrow Y$ is the unique cubic mapping satisfying

$$
\|f(2 x)-2 f(x)-C(x)\|_{Y} \leq \frac{1}{|8|} \tilde{\varphi}_{c}(x)
$$

for all $x \in X$, where

$$
\tilde{\varphi}_{c}(x):=\max \left\{|2| M_{c}(x, x),|2| M_{c}(0, x), M_{c}(x, 2 x)\right\} .
$$

Proof. Similar to the proof of Theorem 3.1 we have

$$
\|f(4 x)-10 f(2 x)+16 f(x)\|_{Y} \leq \psi_{c}(x),
$$

for all $x \in X$, where $\psi_{c}(x):=\max \left\{|2| \varphi_{a}(x, x),|2| \varphi_{a}(0, x), \varphi_{a}(x, 2 x)\right\}$. Let $h: X \rightarrow Y$ be a mapping defined by $h(x):=f(2 x)-2 f(x)$ for all $x \in X$. Therefore (3.29) means that

$$
\|h(2 x)-8 h(x)\|_{Y} \leq \psi_{c}(x)
$$

for all $x \in X$. By the relations (3.24) and (3.25), we infer that

$$
\lim _{n \rightarrow \infty} \max \left\{\frac{\psi_{c}\left(2^{i} x\right)}{|8|^{i}}: \quad 0 \leq i<n\right\}<\infty, \quad \lim _{n \rightarrow \infty} \frac{1}{|8|^{n}} \psi_{c}\left(2^{n} x\right)=0
$$


for all $x \in X$. Replacing $x$ by $2^{n} x$ in (3.30) and dividing both sides of (3.30) by $|8|^{n+1}$ we get

$$
\left\|\frac{1}{8^{n+1}} h\left(2^{n+1} x\right)-\frac{1}{8^{n}} h\left(2^{n} x\right)\right\|_{Y} \leq \frac{1}{|8|^{n+1}} \psi_{c}\left(2^{n} x\right)
$$

for all $x \in X$ and all non-negative integer $n$. So we have

$$
\left\|\frac{1}{8^{n}} h\left(2^{n} x\right)-\frac{1}{8^{m}} h\left(2^{m} x\right)\right\|_{Y} \leq \frac{1}{|8|} \max \left\{\frac{\psi_{c}\left(2^{i} x\right)}{|8|^{i}} m \leq i<n\right\},
$$

for all $x \in X$ and all non-negative integers $n$ and $m$ with $n \geq m$. Therefore we conclude from (3.31) and (3.32) that the sequence $\left\{\frac{1}{8^{n}} h\left(2^{n} x\right)\right\}$ is a Cauchy sequence in $Y$ for all $x \in X$. The sequence $\left\{\frac{1}{8^{n}} h\left(2^{n} x\right)\right\}$ converges in $Y$, for all $x \in X$, since $Y$ is complete. So one can define the mapping $C: X \rightarrow Y$ by

$$
C(x):=\lim _{n \rightarrow \infty} \frac{1}{8^{n}} h\left(2^{n} x\right)
$$

for all $x \in X$. Letting $m=0$ and passing to the limit when $n \rightarrow \infty$ in (3.33), we get (3.28). Now we show that $C$ is a cubic mapping. It follows from (3.31), (3.32) and (3.34) that

$$
\begin{aligned}
\|C(2 x)-8 C(x)\|_{Y} & =\lim _{n \rightarrow \infty}\left\|\frac{1}{8^{n}} h\left(2^{n+1} x\right)-\frac{1}{8^{n-1}} h\left(2^{n} x\right)\right\|_{Y} \\
& \leq \lim _{n \rightarrow \infty} \frac{1}{|8|^{n}} \psi_{c}\left(2^{n} x\right)=0,
\end{aligned}
$$

for all $x \in X$. So

$$
C(2 x)=8 C(x),
$$

for all $x \in X$. On the other hand it follows from (3.24), (3.27) and (3.34) that

$$
\begin{aligned}
\|D C(x, y)\|_{Y} & =\lim _{n \rightarrow \infty} \frac{1}{|8|^{n}}\left\|D h\left(2^{n} x, 2^{n} y\right)\right\|_{Y} \\
& =\lim _{n \rightarrow \infty} \frac{1}{|8|^{n}}\left\{\left\|D f\left(2^{n+1} x, 2^{n+1} y\right)-2 D f\left(2^{n} x, 2^{n} y\right)\right\|_{Y}\right\} \\
& \leq \lim _{n \rightarrow \infty} \frac{1}{|8|^{n}} \max \left\{\left\|D f\left(2^{n+1} x, 2^{n+1} y\right)\right\|_{Y},|2|\left\|D f\left(2^{n} x, 2^{n} y\right)\right\|_{Y}\right\} \\
& \leq \lim _{n \rightarrow \infty} \frac{1}{|8|^{n}} \max \left\{\varphi_{c}\left(2^{n+1} x, 2^{n+1} y\right),|2| \varphi_{c}\left(2^{n} x, 2^{n} y\right)\right\}=0
\end{aligned}
$$

for all $x, y \in X$. Hence the mapping $C$ satisfies (1.2). So by Lemma (1.2), the mapping $x \mapsto C(2 x)-2 C(x)$ is cubic. Therefore (3.35) implies that $C$ is cubic.

To prove the uniqueness of $C$, let $T: X \rightarrow Y$ be another cubic mapping satisfying (3.28). So it follows from (3.26) that $\lim _{t \rightarrow \infty} \frac{\tilde{\varphi}_{c}\left(2^{t x}\right)}{|8|^{t}}=0$, for all $x, y \in X$ and $x \in$ $\left\{0, y, \frac{y}{2}\right\}$. So by relations (3.28) and (3.24)

$$
\|C(x)-T(x)\|_{Y}=\lim _{n \rightarrow \infty} \frac{1}{|8|^{n}}\left\|h\left(2^{n} x\right)-T\left(2^{n} x\right)\right\|_{Y} \leq \frac{1}{|8|} \lim _{n \rightarrow \infty} \frac{1}{|8|^{n}} \tilde{\varphi}_{c}\left(2^{n x}\right)=0,
$$

for all $x \in X$. So $C=T$.

3.6. Theorem. Let $\varphi_{c}: X \times X \rightarrow[0, \infty)$ be a mapping such that

$$
\begin{aligned}
& \lim _{n \rightarrow \infty}|8|^{n} \varphi_{c}\left(\frac{x}{2^{n}}, \frac{y}{2^{n}}\right)=0 \\
& M_{c}(x, y)=\lim _{n \rightarrow \infty} \max \left\{|8|^{i} \varphi_{c}\left(\frac{x}{2^{i}}, \frac{y}{2^{i}}\right): 1 \leq i<n\right\}<\infty \\
& \lim _{t \rightarrow \infty} \lim _{n \rightarrow \infty} \max \left\{|8|^{i} \varphi_{c}\left(\frac{x}{2^{i}}, \frac{y}{2^{i}}\right): t+1 \leq i<t+n\right\}=0
\end{aligned}
$$


for all $y \in X$ and all $x \in\left\{0, y, \frac{y}{2}\right\}$. Suppose that a mapping $f: X \rightarrow Y$ with $f(0)=0$ satisfies the inequality

$$
\|D f(x, y)\|_{Y} \leq \varphi_{c}(x, y)
$$

for all $x, y \in X$. Then the limit

$$
C(x)=\lim _{n \rightarrow \infty} 8^{n}\left[f\left(\frac{x}{2^{n-1}}\right)-2 f\left(\frac{x}{2^{n}}\right)\right]
$$

exists, for all $x \in X$, and the mapping $C: X \rightarrow Y$ is the unique cubic mapping satisfying

$$
\|f(2 x)-2 f(x)-C(x)\|_{Y} \leq \frac{1}{|8|} \tilde{\varphi}_{c}(x),
$$

for all $x \in X$, where

$$
\tilde{\varphi}_{c}(x):=\max \left\{|2| M_{c}(x, x),|2| M_{c}(0, x), M_{c}(x, 2 x)\right\} .
$$

Proof. Let $h: X \rightarrow Y$ be a mapping defined by $h(x):=f(2 x)-2 f(x)$ and let

$$
\psi_{c}(x):=\max \left\{|2| \varphi_{a}(x, x),|2| \varphi_{c}(0, x), \varphi_{c}(x, 2 x)\right\}
$$

for any $x \in X$. Similar to the proof of Theorem 3.5, for every $x \in X$, we have

$$
\|h(2 x)-8 h(x)\|_{Y} \leq \psi_{c}(x) .
$$

From (3.36) and (3.37) we infer that

$$
\lim _{n \rightarrow \infty}|8|^{n} \psi_{c}\left(\frac{x}{2^{n}}\right)=0 \text {, and } \lim _{n \rightarrow \infty} \max \left\{|8|^{i} \psi_{c}\left(\frac{x}{2^{i}}\right): 1 \leq i<n\right\}<\infty,
$$

for all $x \in X$. Replacing $x$ by $\frac{x}{2^{n+1}}$ in (3.40) and multiplying both sides of (3.40) by $|8|^{n}$, we get

$$
\left\|8^{n+1} h\left(\frac{x}{2^{n+1}}\right)-8^{n} h\left(\frac{x}{2^{n}}\right)\right\|_{y} \leq|8|^{n} \psi_{c}\left(\frac{x}{2^{n+1}}\right)
$$

for any $x \in X$ and all non-negative integer $n$. Thus we have

$$
\left\|8^{n} h\left(\frac{x}{2^{n}}\right)-8^{m} h\left(\frac{x}{2^{m}}\right)\right\|_{Y} \leq \frac{1}{|8|} \max \left\{|8|^{i+1} \psi_{c}\left(\frac{x}{2^{i+1}}\right): m \leq i<n\right\},
$$

for all $x \in X$ and all non-negative integers $n$ and $m$ with $n \geq m$. Therefore we conclude from (3.41) and (3.42) that the sequence $\left\{8^{n} h\left(\frac{x}{2^{n}}\right\}\right.$ is a Cauchy sequence in $Y$ for all $x \in X$. Hence the sequence $\left\{8^{n} h\left(\frac{x}{2^{n}}\right\}\right.$ converges in $Y$, for all $x \in X$, since $Y$ is complete. So one can define the mapping $C: X \rightarrow Y$ by

$$
C(x):=\lim _{n \rightarrow \infty} 8^{n} h\left(\frac{x}{2^{n}}\right)
$$

for all $x \in X$. Letting $m=0$ and passing to the limit when $n \rightarrow \infty$ in (3.43) we get (3.39). The rest of the proof is similar to the proof of Theorem 3.5.

The following two corollaries follow from Theorems 3.5 and 3.6.

3.7. Corollary. Let $\theta, r, s$ be non-negative real numbers such that $r, s>3$ or $0 \leq r, s<3$ and $|2|<1$. Suppose that a function $f: X \rightarrow Y$ with $f(0)=0$ satisfies the inequality of Corollary 3.3, for all $x, y \in X$. Then there exists a unique cubic mapping $C: X \rightarrow Y$ satisfying

$$
\begin{array}{r}
\|f(2 x)-2 f(x)-C(x)\|_{Y} \\
\leq \frac{\theta}{|8|} \begin{cases}1, & r=s=0 \\
\|x\|_{X}^{r}, & r>0, s=0 \\
|2|\|x\|_{X}^{s}, & r=0, s>0 \\
\max \left\{|2|\left(\|x\|_{X}^{r}+\|x\|_{X}^{s}\right),\left(\|x\|_{X}^{r}+\|2 x\|_{X}^{s}\right),\right. & r, s>0,\end{cases}
\end{array}
$$


for all $x \in X$ when $r, s>3$ and satisfying

$$
\begin{aligned}
\|f(2 x)-2 f(x)-C(x)\|_{Y} & \\
& \leq \theta \begin{cases}1, & r=s=0 \\
\frac{\|x\|_{X}^{r}}{|2|^{r}}, & r>0, s=0 \\
\frac{\|x\|_{X}^{s}}{|2|^{s}} \max \left\{|2|,|2|^{s}\right\}, & r=0, s>0 \\
\max \left\{|2|\left(\frac{\|x\|_{X}^{r}}{|2|^{r}}+\frac{\|x\|_{X}^{s}}{|2|^{s}}\right),\left(\frac{\|x\|_{X}^{r}}{|2|^{r}}+\|x\|_{X}^{s}\right),\right. & r, s>0,\end{cases}
\end{aligned}
$$

for all $x \in X$ when $r, s<3$.

3.8. Corollary. Let $\theta \geq 0$ and $r, s>0$ be non-negative real numbers such that $\lambda:=$ $r+s \neq 3$ and $|2|<1$. Suppose that the function $f: X \rightarrow Y$ with $f(0)=0$ satisfies the inequality (3.23), for all $x, y \in X$. Put $W=\max \left\{|2|,|2|^{s}\right\}$. Then there exists a unique cubic mapping $C: X \rightarrow Y$ satisfying the inequality

$$
\|f(2 x)-2 f(x)-C(x)\|_{Y} \leq \frac{1}{|8|} W \theta\|x\|_{X}^{r}\|x\|_{Y}^{s},
$$

for all $x \in X$, when $\lambda>3$ and satisfying

$$
\|f(2 x)-2 f(x)-C(x)\|_{Y} \leq \frac{1}{|2|^{\lambda}} W \theta\|x\|_{X}^{r}\|x\|_{Y}^{s}
$$

for all $x \in X$, when $\lambda<3$.

3.9. Theorem. Let $\varphi: X \times X \rightarrow[0, \infty)$ be a mapping such that

$$
\begin{aligned}
& \lim _{n \rightarrow \infty} \frac{1}{|8|^{n}} \varphi\left(2^{n} x, 2^{n} y\right)=0 \\
& M_{c}(x, y)=\lim _{n \rightarrow \infty} \max \left\{\frac{1}{|8|^{i}} \varphi\left(2^{i} x, 2^{i} y\right): 0 \leq i<n\right\}<\infty \\
& \lim _{t \rightarrow \infty} \lim _{n \rightarrow \infty} \max \left\{\frac{1}{|8|^{i}} \varphi\left(2^{i} x, 2^{i} y\right): \quad t \leq i<t+n\right\}=0
\end{aligned}
$$

for all $y \in X$ and all $x \in\left\{0, y, \frac{y}{2}\right\}$. Suppose that a mapping $f: X \rightarrow Y$ with $f(0)=0$ satisfies the inequality

$$
\|D f(x, y)\|_{Y} \leq \varphi(x, y), \quad x, y \in X .
$$

Then there exist a unique additive mapping $A: X \rightarrow Y$ and a unique cubic mapping $C: X \rightarrow Y$ such that for every $x \in X$,

$$
\|f(x)-A(x)-C(x)\|_{Y} \leq \frac{1}{|48|} \max \left\{|4| \tilde{\varphi}_{a}(x), \tilde{\varphi}_{c}(x)\right\}
$$

where

$$
\begin{aligned}
M_{a}(x, y) & :=\lim _{n \rightarrow \infty} \max \left\{\frac{1}{|2|^{i}} \varphi\left(2^{i} x, 2^{i} y\right) 0 \leq i<n\right\} \\
\tilde{\varphi}_{a}(x) & :=\max \left\{|2| M_{a}(x, x),|2| M_{a}(0, x), M_{a}(x, 2 x)\right\} \\
\tilde{\varphi}_{c}(x) & :=\max \left\{|2| M_{c}(x, x),|2| M_{c}(0, x) . M_{c}(x, 2 x)\right\}
\end{aligned}
$$

Proof. By Theorems 3.1 and 3.5, there exist an additive mapping $A_{0}: X \rightarrow Y$ and a cubic mapping $C_{0}: X \rightarrow Y$ such that

$$
\left\|f(2 x)-8 f(x)-A_{0}(x)\right\|_{Y} \leq \frac{1}{|2|} \tilde{\varphi}_{a}(x), \quad\left\|f(2 x)-2 f(x)-C_{0}(x)\right\|_{Y} \leq \frac{1}{|8|} \tilde{\varphi}_{c}(x)
$$

for all $x \in X$. This implies that for any $x \in X$,

$$
\left\|f(x)+\frac{1}{6} A_{0}(x)-\frac{1}{6} C_{0}(x)\right\|_{Y} \leq \frac{1}{|48|} \max \left\{|4| \tilde{\varphi}_{a}(x), \tilde{\varphi}_{c}(x)\right\} .
$$


So we obtain (3.44), by letting $A(x)=\frac{1}{6} A_{0}(x)$ and $C(x)=\frac{1}{6} C_{0}(x)$, for all $x \in X$.

To prove the uniqueness of $A$ and $C$, let $A_{1}, C_{1}: X \rightarrow Y$ be other additive and cubic mappings satisfying (3.44).

Put $A^{\prime}=A-A_{1}$ and $C^{\prime}=C-C_{1}$. So

$$
\begin{aligned}
\left\|A^{\prime}(x)+C^{\prime}(x)\right\|_{Y} & \leq \max \left\{\|f(x)-A(x)-C(x)\|_{Y},\left\|f(x)-A_{1}(x)-C_{1}(x)\right\|_{Y}\right\} \\
& \leq \frac{1}{|48|} \max \left\{|4| \tilde{\varphi}_{a}(x), \tilde{\varphi}_{c}(x)\right\}
\end{aligned}
$$

for all $x \in X$. The fact that for every $x \in X$,

$$
\lim _{n \rightarrow \infty} \frac{1}{|8|^{n}} \tilde{\varphi}_{c}\left(2^{n} x\right)=\lim _{n \rightarrow \infty} \frac{1}{|2|^{n}} \tilde{\varphi}_{a}\left(2^{n} x\right)=0
$$

and (3.45) imply that

$$
\lim _{n \rightarrow \infty} \frac{1}{|2|^{n}}\left\|A^{\prime}\left(2^{n} x\right)+C^{\prime}\left(2^{n} x\right)\right\|_{Y}=0
$$

for all $x \in X$. Therefore $A^{\prime}=0$. So it follows from (3.45) that

$$
\left\|C^{\prime}(x)\right\|_{Y}=\lim _{n \rightarrow \infty}\left\|\frac{C^{\prime}\left(2^{n} x\right)}{8^{n}}\right\|_{Y} \leq \lim _{n \rightarrow \infty} \frac{1}{|4|} \max \left\{|4| \frac{\tilde{\varphi}_{a}(x)}{|8|^{n}}, \frac{\tilde{\varphi}_{c}(x)}{|8|^{n}}\right\},
$$

for all $x \in X$. Therefore $C^{\prime}=0$.

The next theorem is an alternative result of Theorem 3.9.

3.10. Theorem. Let $\varphi: X \times X \rightarrow[0, \infty)$ be a mapping such that

$$
\begin{aligned}
& \lim _{n \rightarrow \infty}|2|^{n} \varphi\left(\frac{x}{2^{n}}, \frac{y}{2^{n}}\right)=0 \\
& M_{a}(x, y):=\lim _{n \rightarrow \infty} \max \left\{|2|^{i} \varphi\left(\frac{x}{2^{i}}, \frac{y}{2^{i}}\right): 1 \leq i<n\right\}<\infty \\
& \lim _{t \rightarrow \infty} \lim _{n \rightarrow \infty} \max \left\{|2|^{i} \varphi\left(\frac{x}{2^{i}}, \frac{y}{2^{i}}\right): t+1 \leq i<t+n\right\}=0,
\end{aligned}
$$

for all $y \in X$ and all $x \in\left\{0, y, \frac{y}{2}\right\}$. Suppose that a mapping $f: X \rightarrow Y$ with $f(0)=0$ satisfies the inequality

$$
\|D f(x, y)\|_{Y} \leq \varphi(x, y)
$$

for all $x, y \in X$. Then there exist a unique additive mapping $A: X \rightarrow Y$ and a unique cubic mapping $C: X \rightarrow Y$ such that

$$
\|f(x)-A(x)-C(x)\|_{Y} \leq \frac{1}{|48|} \max \left\{|4| \tilde{\varphi}_{a}(x), \tilde{\varphi}_{c}(x)\right\}, \quad x \in X,
$$

where

$$
\begin{aligned}
& M_{c}(x, y):=\lim _{n \rightarrow \infty} \max \left\{|8|^{i} \varphi\left(\frac{x}{2^{i}}, \frac{y}{2^{i}}\right): 1 \leq i<n\right\}, \\
& \tilde{\varphi}_{a}(x):=\max \left\{|2| M_{a}(x, x),|2| M_{a}(0, x), M_{a}(x, 2 x)\right\} \\
& \tilde{\varphi}_{c}(x):=\max \left\{|2| M_{c}(x, x),|2| M_{c}(0, x), M_{c}(x, 2 x)\right\}
\end{aligned}
$$

for all $x \in X$.

3.11. Corollary. Let $r, s, \theta$ be non-negative real numbers such that $r, s>3$ or $0 \leq r, s<1$ and $|2|<1$. Suppose that a function $f: X \rightarrow Y$ with $f(0)=0$ satisfies the inequality Corollary 3.3, for all $x, y \in X$. Then there exist a unique additive mapping $A: X \rightarrow Y$ and a unique cubic mapping $C: X \rightarrow Y$ such that

$$
\|f(x)-A(x)-C(x)\|_{Y} \leq \frac{1}{|48|} \gamma_{a 1} \text { for all } x \in X \text { when } 0 \leq r, s<1
$$


and

$$
\|f(x)-A(x)-C(x)\|_{Y} \leq \frac{1}{|48|} \max \left\{\gamma_{a 2}, \gamma_{c}\right\} \text { for all } x \in X \text { when } r, s>3
$$

where

$$
\begin{gathered}
\gamma_{a 1}= \begin{cases}|8| \theta, & r=s=0 \\
\frac{|8| \theta\|x\|_{X}^{r}}{|2|^{r}}, & r>0, s=0 \\
\frac{|8||2|^{s} \theta\|x\|_{X}^{s}}{|2|^{s}}, & r=0, s>0 \\
|8| \theta\left(\frac{\|x\|_{X}^{r}}{|2|^{r}}+\|x\|_{X}^{s}\right), & r, s>0,\end{cases} \\
\gamma_{a 2}= \begin{cases}|4| \theta, & r=s=0 \\
|4| \theta\|x\|_{X}^{r}, & r>0, s=0 \\
|8| \theta\|x\|_{X}^{s}, & r=0, s>0 \\
|4| \theta \max \left\{|2|\left(\|x\|_{X}^{r}+\|x\|_{X}^{s}\right),\left(\|x\|_{X}^{r}+\|2 x\|_{X}^{s}\right),\right. & r, s>0,\end{cases} \\
\gamma_{c}= \begin{cases}|8| \theta, \quad r=s=0 \\
\frac{|8| \theta\left\|_{x}\right\|_{X}^{r}}{|2|^{r}}, & r>0, s=0 \\
\frac{|8| \theta \mid \|_{X}^{s}}{|2|^{s}} \max \left\{|2|^{s},|2|\right\}, & r=0, s>0 \\
|8| \theta \max \left\{|2|\left(\frac{\|x\|_{X}^{r}}{|2|^{r}}+\frac{\|x\|_{X}^{s}}{|2|^{s}}\right),\left(\frac{\|x\|_{X}^{r}}{|2|^{r}}+\|x\|_{X}^{s}\right)\right\}, & r, s>0 .\end{cases}
\end{gathered}
$$

3.12. Corollary. Let $\theta \geq 0$ and $r, s>0$ be real numbers such that $\lambda:=r+s \in$ $(0,1) \bigcup(3,+\infty)$ and $|2|<\overline{1}$. Suppose that a function $f: X \rightarrow Y$ with $f(0)=0$ satisfies the inequality (3.23) for all $x, y \in X$. Then there exist a unique additive mapping $A$ : $X \rightarrow Y$ and a unique cubic mapping $C: X \rightarrow Y$ such that

$$
\|f(x)-A(x)-C(x)\|_{Y} \leq \frac{1}{|48|} W \theta\|x\|_{X}^{r}\|x\|_{Y}^{s} \text { for all } x \in X \text { and } \lambda>3,
$$

where $W=\max \left\{|2|,|2|^{s}\right\}$

$$
\|f(x)-A(x)-C(x)\|_{Y} \leq \frac{|2|^{s}}{|6||2|^{\lambda}} \theta\|x\|_{X}^{r}\|x\|_{Y}^{s} \text { for all } x \in X \text { and } 0<\lambda<1 .
$$

3.13. Theorem. Let $\varphi: X \times X \rightarrow[0, \infty)$ be a mapping such that

$$
\begin{aligned}
& \lim _{n \rightarrow \infty} \frac{1}{|2|^{n}} \varphi\left(2^{n} x, 2^{n} y\right)=0, \\
& M_{a}(x, y):=\lim _{n \rightarrow \infty} \max \left\{\frac{1}{|2|^{i}} \varphi\left(2^{i} x, 2^{i} y\right): 0 \leq i<n\right\}<\infty, \\
& \lim _{t \rightarrow \infty} \lim _{n \rightarrow \infty} \max \left\{\frac{1}{|2|^{i}} \varphi\left(2^{i} x, 2^{i} y\right): \quad t \leq i<t+n\right\}=0,
\end{aligned}
$$

for all $y \in X$ and all $x \in\left\{0, y, \frac{y}{2}\right\}$. Also suppose

$$
\begin{aligned}
& \lim _{n \rightarrow \infty}|8|^{n} \varphi\left(\frac{x}{2^{n}}, \frac{y}{2^{n}}\right)=0, \\
& M_{c}(x, y):=\lim _{n \rightarrow \infty} \max \left\{|8|^{i} \varphi\left(\frac{x}{2^{i}}, \frac{y}{2^{i}}\right): 1 \leq i<n\right\}<\infty, \\
& \lim _{t \rightarrow \infty} \lim _{n \rightarrow \infty} \max \left\{|8|^{i} \varphi\left(\frac{x}{2^{i}}, \frac{y}{2^{i}}\right): t+1 \leq i<t+n\right\}=0,
\end{aligned}
$$

for all $x, y \in X$ and all $x \in\left\{0, y, \frac{y}{2}\right\}$. Suppose that a mapping $f: X \rightarrow Y$ with $f(0)=0$ satisfies the inequality

$$
\|D f(x, y)\|_{Y} \leq \varphi(x, y)
$$


for all $x, y \in X$. Then there exist a unique additive mapping $A: X \rightarrow Y$ and a unique cubic mapping $C: X \rightarrow Y$ such that

$$
\|f(x)-A(x)-C(x)\|_{Y} \leq \frac{1}{|48|} \max \left\{|4| \tilde{\varphi}_{a}(x), \tilde{\varphi}_{c}(x)\right\}
$$

where

$$
\begin{aligned}
& \tilde{\varphi}_{a}(x):=\max \left\{|2| M_{a}(x, x),|2| M_{a}(0, x), M_{a}(x, 2 x)\right\} \\
& \tilde{\varphi}_{c}(x):=\max \left\{|2| M_{c}(x, x),|2| M_{c}(0, x), M_{c}(x, 2 x)\right\}
\end{aligned}
$$

for all $x \in X$.

Proof. The proof is similar to the proof of Theorem 3.9.

3.14. Corollary. Let $\theta, r, s$ be non-negative real numbers such that $1<r, s<3$ and $|2|<1$. Suppose that a function $f: X \rightarrow Y$ with $f(0)=0$ satisfies the inequality $\|D f(x, y)\|_{Y} \leq \theta\left(\|x\|_{X}^{r}+\|y\|_{X}^{s}\right)$ for all $x, y \in X$. Then there exist a unique additive mapping $A: X \rightarrow Y$ and a unique cubic mapping $C: X \rightarrow Y$ such that

$$
\|f(x)-A(x)-C(x)\|_{Y} \leq \frac{1}{|48|} \max \left\{|4| \gamma_{a}(x), \gamma_{c}(x)\right\} \text { for all } x \in X,
$$

where

$$
\begin{aligned}
& \gamma_{a}(x)=\max \left\{|2| \theta\left(\|x\|_{X}^{r}+\|x\|_{X}^{s}\right), \theta\left(\|x\|_{X}^{r}+\|2 x\|_{X}^{s}\right)\right\}, \\
& \gamma_{c}(x)=|8| \theta \max \left\{|2|\left(\frac{\|x\|_{X}^{r}}{|2|^{r}}+\frac{\|x\|_{X}^{s}}{|2|^{s}}\right),\left(\frac{\|x\|_{X}^{r}}{|2|^{r}}+\|x\|_{X}^{s}\right) .\right.
\end{aligned}
$$

3.15. Corollary. Let $\theta, r, s$ be non-negative real numbers such that $1<\lambda:=r+s<3$. Suppose that a function $f: X \rightarrow Y$ with $f(0)=0$ satisfies the inequality

$$
\|D f(x, y)\|_{Y} \leq \begin{cases}\theta\|x\|_{X}^{r}, & r>0, s=0 \\ \theta\|y\|_{X}^{s}, & r=0, s>0 \\ \theta\|x\|_{X}^{r}\|y\|_{X}^{s}, & r, s>0\end{cases}
$$

for all $x, y \in X$. Then there exist a unique additive mapping $A: X \rightarrow Y$ and a unique cubic mapping $C: X \rightarrow Y$ such that

$$
\|f(x)-A(x)-C(x)\|_{Y} \leq \frac{1}{|48|} \max \left\{|4| \gamma_{a}(x), \gamma_{c}(x)\right\} \text { for all } x \in X
$$

where,

$$
\begin{gathered}
\gamma_{a}(x)= \begin{cases}\theta\|x\|_{X}^{r}, & r>0, s=0 \\
|2| \theta\left\|_{x}\right\|_{X}^{s}, & r=0, s>0 \\
\theta\|x\|_{X}^{\lambda} \max \left\{|2|,|2|^{s}\right\}, & r, s>0,\end{cases} \\
\gamma_{c}(x)= \begin{cases}\frac{|8| \theta\|x\|_{X}^{r}}{|2|^{r}}, & r>0, s=0 \\
\frac{|8| \theta \mid x \|_{X}^{s}}{||^{\mid}} \max \left\{|2|,|2|^{s}\right\}, & r=0, s>0 \\
\frac{|8| \theta\|x\|_{X}^{\lambda}}{|2|^{\lambda}} \max \left\{|2|,|2|^{s}\right\}, & r, s>0 .\end{cases}
\end{gathered}
$$

3.16. Remark. The hypothesis $f(0)=0$ is not essential in the statement of the theorems, since it is possible to deal with the auxiliary function $g(x):=f(x)-f(0)$ for which we have $D g(x, y)=D f(x, y)$. 


\section{References}

[1] T. Aoki, On the stability of the linear transformation in Banach spaces, J. Math. Soc. Japan 2, 64-66, 1950.

[2] L. M. Arriola and W. A. Beyer, Stability of the Cauchy functional equation over p-adic fields, Real Anal. Exchange 31, 125-132, 2055/2006.

[3] C. Baak, S. -K. Hong, M. -J. Kim, Generalized quadratic mappings of r-type in several variables, J. Math. Anal. Appl. 310, 116-127, 2005.

[4] J. -H. Bae and W. -G. Park, A functional equation originating from quadratic forms, J. Math. Anal. Appl. 326, 1142-1148, 2007.

[5] D. G. Bourgin, Classes of transformations and bordering transformations, Bull. Amer. Math. Soc. 57, 223-237, 1951.

[6] S. Czerwik, Stability of Functional Equations of Ulam-Hyers-Rassias Type, (Hadronic Press, Palm Harbor, Florida, 2003).

[7] G. L. Forti, Hyers-Ulam stability of functional equations in several variables Aequationes Math. 50 (1-2), 143-190, 1995.

[8] D. H. Hyers, On the stability of the linear functional equation, Proc. Nat. Acad. Sci. U. S. A. 27, 222-224, 1941.

[9] D. H. Hyers and Th. M. Rassias, Approximate homomorphisms, Aequationes Math. 44, 125-153, 1992.

[10] D. H. Hyers, G. Isac and Th. M. Rassias, Stability of Functional Equations in Several Variables, (Birkhäuser, Basel, 1998).

[11] S.-M. Jung, On the Hyers-Ulam Stability of the Functional Equations That Have the Quadratic Property, Journal of Mathematical Analysis and Applications 222, 126-137, 1998.

[12] S.-M. Jung, On the Hyers-Ulam-Rassias Stability of a Quadratic Functional Equation, Journal of Mathematical Analysis and Applications 232, 384-393, 1999.

[13] S. -M. Jung, Hyers-Ulam-Rassias Stability of Functional Equations in Mathematical Analysis, (Hadronic Press lnc., Palm Harbor, Florida, 2001).

[14] G. H. Kim, On the Hyers-Ulam-Rassias stability of functional equations in n-variables, J. Math. Anal. Appl. 299, 375-391, 2004.

[15] M. S. Moslehian and Th. M. Rassias, Stability of functional equations in non-Archimedian spaces, Appl. Anal. Disc. Math. 1, 325-334, 2007.

[16] M. S. Moslehian, and Gh. Sadeghi, Stability of two type of cubic functional equations in non-Archimedian spaces, Real. Anal. Exchange, 33 (2), 375-383, 2008.

[17] A. Najati, and G. Z. Eskandani, Stability of a mixed additive and cubic functional equation in quasi-Banach spaces, J. Math. Anal. Appl. 342, 1318-1331, 2008.

[18] W. -G. Park, J. -H. Bae, On a bi-quadratic functional equation and its stability, Nonlinear Analysis 6 No.2, 643-654, 2005.

[19] C. G. Park, Generalized quadratic mappings in several variables, Nonlinear Analysis 57, 713-722, 2004.

[20] D. Popa and I. Rasa, The Frechet functional equation with application to the stability of certain operators, J. Approx. Theory 164, 138-144, 2012.

[21] D. Popa and I. Rasa, On the Hyers-Ulam stability of the linear differential equation, J. Math. Anal. Appl. 381, 530-537, 2011.

[22] Th. M. Rassias, Functional Equations, Inequalities and Applications, (Kluwer Academic Publishers, Dordrecht, Boston and London, 2003).

[23] Th. M. Rassias, On the stability of functional equations and a problem of Ulam, Acta Appl. Math. 62 (1), 23-130, 2000.

[24] Th. M. Rassias, On the stability of the linear mapping in Banach spaces, Proc. Amer. Math. Soc. 72, 297-300, 1978.

[25] T. Xu, J.M. Rassias ,W. Xu, A fixed point approach to the stability of a general mixed additive-cubic equation on Banach modules, Acta Mathematica Scientia 32, 866-892, 2012.

[26] S. M. Ulam, Problems in Modern Mathematics, (Chapter VI, Science Editions, Wiley, New York, 1960). 